\title{
The effectiveness of silver diamine fluoride in arresting caries
}

\author{
Abstracted from \\ Gao SS, Zhao IS, Hiraishi N, Duangthip D, Mei ML, Lo ECM, Chu CH. \\ Clinical trials of silver diamine fluoride in arresting caries among children. A systematic review. JDR Clin Trans Res 2016; 1: 201-210. \\ Address for correspondence: Chun-Hung Chu, Faculty of Dentistry, The University of Hong Kong, \\ 34 Hospital Road, Hong Kong, China. E-mail: chchu@hku.hk
}

\section{Question: Is silver diamine fluoride (SDF) effective in reducing caries in children?}

Data sources PubMed, Embase, Scopus, China National Knowledge Infrastructure (CNKI), Ichushi-web, Biblioteca Virtual en Salud Espana (BVSE) and Biblioteca Virtual em Saude (BVS) databases. There were no limits on language or publication dates.

Study selection Two reviewers selected prospective clinical studies investigating SDF treatment for caries prevention in children.

Data extraction and synthesis Data was abstracted independently by two reviewers and risk of bias assessed. Meta-analysis was performed on studies in which the caries-arresting rate using 38\% SDF solution on primary teeth could be obtained or calculated.

Results Nineteen studies were included; 16 were conducted in the primary dentition and three in permanent dentition. Fourteen studies used 38\% SDF, three 30\% SDF, and two 10\% SDF. Eight studies using $38 \%$ SDF contributed to a meta-analysis and the overall proportion of arrested caries was $81 \%(95 \% \mathrm{Cl} ; 68-89 \%)$. Percentage reductions were also calculated for $6,12,18,24$ and $>30$ months. Arrested carious lesions stained black but no other adverse effects were reported. Conclusions SDF commonly used at a high concentration (38\%, $44,800 \mathrm{ppm}$ fluoride) is effective in arresting caries among children. There is no consensus on its number and frequency of application to arrest caries. Further studies are necessary to develop evidence-based guidelines on its use in children.

\section{Commentary}

Childhood caries remains a significant worldwide public health problem with untreated decay in primary teeth being the tenth most prevalent condition with untreated caries in permanent teeth being the commonest. ${ }^{1}$ Traditional restorative treatment approaches are expensive and are not available to all so costeffective prevention or management approaches are needed. Silver diamine fluoride has been in active use in many countries and the first review of its effectiveness in $2009^{2}$ suggested caries reductions ranging between $70-96 \%$. The aim of this current review was to look at the latest evidence on the effectiveness of SDF in reducing caries in children.

An extensive database search had been conducted with no restrictions on publication date or language so the relevant studies should have been identified. The authors have chosen to include all prospective studies not restricting inclusion to randomised controlled trials so many of the included studies have no control group. Of those studies included in the meta-analysis only five of the eight had control groups. The authors have undertaken a risk of bias assessment using the Cochrane tool, although, it is not clear if this was conducted independently. It is not specifically stated how many of the included studies were randomised controlled trials and the random sequence generation was only considered to be low risk in four studies and none of the studies were considered to be low risk for allocation concealment. Given that SDF is known to cause black staining of the teeth it is interesting to see that five of the studies are rated as being at low risk of detection bias as this would be difficult to achieve.

These concerns would suggest that the quality of the available evidence in this review is likely to be low. However, the included studies have shown consistently high levels of caries arrest and some studies have demonstrated a better effect than fluoride varnish. ${ }^{3,4}$ SDF also offers a low cost alternative to other topical fluoride vehicles. Its main limitation remains the fact that it stains carious lesions black which may not be acceptable to many children and parents. A recent trial ${ }^{5}$ has examined a nano-silver diamine fluoride agent which was able to demonstrate effectiveness in arresting dentine caries but not stain teeth. For both this new SDF agent and the older 38\% SDF solution high quality randomised controlled trials are needed to fully clarify their role in the prevention and management of childhood caries.

\section{Derek Richards}

Department of Public Health, NHS Forth Valley and Centre for Evidence-based Dentistry, Dental Health Services Research Unit, Dundee Dental Hospital and School Dundee University, Dundee

1. Marcenes W, Kassebaum NJ, Bernabé E, Flaxman A, Naghavi M, Lopez A, Murray CJ. Global burden of oral conditions in 1990-2010: a systematic analysis. J Dent Res 2013; 92: 592-597.

2. Rosenblatt A, Stamford TC, Niederman R. Silver diamine fluoride: a caries "silverfluoride bullet". J Dent Res 2009; 88: 16-25.

3. Chu CH, Lo EC, Lin H.. Effectiveness of silver diamine fluoride and sodium fluoride varnish in arresting dentin caries in Chinese pre-school children. J Dent Res 2002 81: 767-770.

4. Duangthip $\mathrm{D}$, $\mathrm{Chu} \mathrm{CH}$, Lo EC. A randomized clinical trial on arresting dentine caries in preschool children by topical uorides-18 month results. J Dent 2016; 44: 57-63.

5. Santos VE Jr, Vasconcelos Filho A, Targino AG, Flores MA, Galembeck A, Caldas AF Jr, Rosenblatt A. A new "silver-bullet" to treat caries in children-nano silver fluoride: a randomised clinical trial. J Dent 2014; 42: 945-951.

Evidence-Based Dentistry (2017) 18, 70. doi:10.1038/sj.ebd.6401250 